\title{
Duration of observation required in detecting fasciculation potentials in amyotrophic lateral sclerosis using high-density surface EMG
}

\author{
Ping Zhou ${ }^{1,2,3^{*}}$, Xiaoyan Li $i^{1}$, Faezeh Jahanmiri-Nezhad ${ }^{1,4}$, William Zev Rymer ${ }^{1,2}$ and Paul E Barkhaus ${ }^{5}$
}

\begin{abstract}
Background: High-density surface electromyography (HD-SEMG) has recently emerged as a potentially useful tool in the evaluation of amyotrophic lateral sclerosis (ALS). This study addresses a practical constraint that arises when applying HD-SEMG for supporting the diagnosis of ALS; specifically, how long the surface EMG should be recorded before one can be confident that fasciculation potentials (FPs) are absent in a muscle being tested.

Methods: HD-SEMG recordings of 29 muscles from 11 ALS patients were analyzed. We used the distribution of intervals between FPs, and estimated the observation duration needed to record from one to five FPs with a probability approaching unity. Such an approach was previously tested by Mills with a concentric needle electrode.

Results: We found that the duration of recording was up to $70 \mathrm{~s}$ in order to record a single FP with a probability approaching unity. Increasing recording time to 2 minutes, the probability of recording five FPs approached approximately 0.95 .
\end{abstract}

Conclusions: HD-SEMG appears to be a suitable method for capturing FPs comparable to intramuscular needle EMG.

\section{Introduction}

Amyotrophic lateral sclerosis (ALS) is a chronic, progressive, degenerative disease of the upper and lower motor neurons that almost always results in reduced life expectancy. To date, no definite biologic marker exists to ascertain the diagnosis. To improve the diagnosis and ascertainment of ALS, Brooks et al. [1] developed consensus criteria using clinical data, imaging studies, and electrophysiological testing (i.e., electromyography [EMG]). While EMG does not make the diagnosis, it is an important adjunct test used to support the diagnosis.

Examination of spontaneous muscle activity is an important part of routine EMG, particularly in supporting the diagnosis of ALS and its variants [2,3]. Spontaneous EMG activity such as fibrillation potentials [2] and fasciculation potentials (FPs) are often seen, although other

\footnotetext{
* Correspondence: p-zhou@northwestern.edu

${ }^{1}$ Sensory Motor Performance Program, Rehabilitation Institute of Chicago,

345 E. Superior St, Suite 1406, Chicago, IL 60611, USA

${ }^{2}$ Department of Physical Medicine and Rehabilitation, Northwestern

University, Chicago, IL, USA

Full list of author information is available at the end of the article
}

types of activity may also be recorded (e.g. complex repetitive discharges, myokymic discharges, neuromyotonic discharges, etc.). While fibrillation potentials are considered to be the sine qua non of active denervation such as seen in ALS, recent discussion has revisited the potential application of FPs to enhance the sensitivity of diagnosis of ALS [3-5]. In all cases of ALS, the routine EMG should also show enlarged, unstable, typically complex motor unit action potentials that represent ongoing reinnervation [6]. Though not unique or specific to ALS, it is this combination of EMG evidence for active denervation associated with evidence of chronic reinnervation that defines ALS electromyographically, in association with clinical criteria.

If new criteria are to be considered in the use of FPs to facilitate the earlier diagnosis of ALS [3,4], then it is reasonable to try to find ways to optimize their detection. During routine EMG examination of a patient for potential ALS, a practical consideration is: how long should we observe this needle electrode recording before concluding that fasciculation potentials are absent? [7]. Mills answered this question by calculating the probability 
values for observing one to five FPs in relation to duration of observation. It was found that using a needle electrode, up to $90 \mathrm{~s}$ may be required to record a single FP with a probability approaching unity while the duration of recording should be $180 \mathrm{~s}$ to observe five FPs [7].

Compared with positive sharp waves or fibrillation potentials that are detected solely by intramuscular needle electrodes, FPs can be recorded by both needle and surface electrodes [8]. In recent years, high-density surface EMG (HD-SEMG) techniques have been developed using electrode arrays comprised of a number of recording probes having minute skin-electrode contact area and small inter-electrode distances [9-12]. Because of the added spatial information, such electrode arrays can offer additional investigative and diagnostic components for examination of the neuromuscular system [13-19].

When clinicians use HD-SEMG to examine a patient with potential ALS, the analogous practical question similar to routine needle electrode EMG recordings is again "how long should the HD-SEMG signal be recorded before the tested muscle can be assumed not to have FPs?". In this short report, we attempt to address this question using Mills' approach by calculating the probability of detecting FPs in relation to observation duration of the HD-SEMG signal [7].

\section{Methods}

Eleven subjects ( 8 males, 3 females, age: $57.4 \pm 8.2$ years, weight: $87.0 \pm 26.6$ kilograms, height: $175.5 \pm 10.9 \mathrm{~cm}$ ) with Definite ALS or Probable ALS with Laboratory Support based on El Escorial criteria [1] participated in this study. All subjects gave written, informed consent prior to their participation. All data recordings were performed at the last author's institution and had the approval of the local Human Studies Committee.

Each subject was positioned comfortably supine on an examination table with a pillow under their head. After placement of the surface electrode array for HD-SEMG signal detection, the subjects were asked to completely relax. The tested arm was placed in its natural, resting position. For the first dorsal interosseous (FDI) and thenar muscles, the hand was typically in semi-pronation. The biceps brachii (BB) was recorded with the elbow partially flexed and forearm in semi-pronation. HDSEMG signals were recorded for at least 3 minutes in a relaxed condition. No feedback was provided to subjects during the recording.

In each experiment, a flexible surface electrode array (Figure 1A, TMS International BV, Enschede, The Netherlands) was used for FDI and thenar recordings. A 20channel bar electrode array (Figure 1B, designed and fabricated in our laboratory) was used for BB recordings. The 2-dimensional flexible array covered the whole surface area of the FDI or thenar muscles while the linear bar electrode array was located from proximal to distal tendon junctions of the $\mathrm{BB}$ muscles. The surface electrode array signals were amplified by the Refa128 EMG Recording System (TMS International BV, Enschede, The Netherlands), with a reference electrode located on the olecranon (each channel also had a common feedback subtraction of the average of all the recording channels). The surface EMG signals were sampled at $2 \mathrm{kHz}$ per channel, with a system band pass filter setting of $20-500 \mathrm{~Hz}$. Before starting the recording, each channel's baseline signal was carefully examined. If a channel's signal quality was poor and further efforts failed to improve the signal, the channel was disconnected. It was typical that 2-5 channels were disconnected during the experiment. The quality of the signal was online monitored during data collection. Since the recording was performed in a resting muscle the signal quality usually remained stable.

The recorded HD-SEMG from the Refa128 system was imported to the Spike 2 software (version 5.12; Cambridge Electronics Design [CED], Cambridge, UK) for display and off-line processing. This software allows viewing or processing of selected channels of the
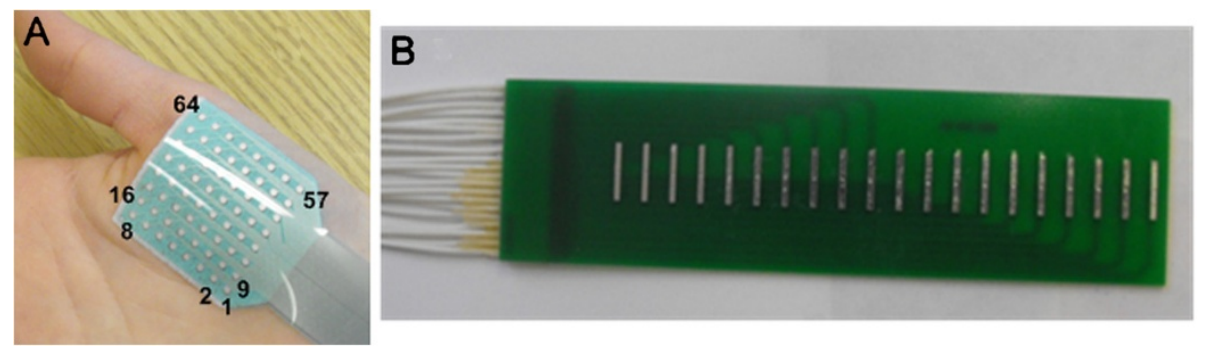

Figure 1 The electrode arrays used for FP recordings. (A) shows a flexible surface electrode array (TMS International BV, Enschede, The Netherlands) that contains 64 recording electrodes arranged in an $8 \times 8$ square matrix. The inter-electrode-distance (IED) between two consecutive recording electrodes in both $(x$ and $y$ ) directions is $4 \mathrm{~mm}$ and each recording electrode has a circular recording surface of $1.2 \mathrm{~mm}$ in diameter. (B) shows our custom made 20-channel bar electrode array. The distance between two consecutive recording bars is $5 \mathrm{~mm}$ and each bar width is $1 \mathrm{~mm}$ in a linear arrangement. 
electrode array and is useful for long duration and multi-channel signal processing. FPs were detected using a threshold-based spike detection algorithm across all the channels. The occurrence time of each FP from the start of the recording was determined, and was then imported to MatLab (Version R2008a, MathWorks, Natick, MA) for further processing.
For each recording, let $t(1), t(2) \ldots t(n), 1 \leq n \leq N$ represent the times of occurrence of FPs, and $N$ is the total number of FPs. The intervals between FPs were calculated [7]:

$$
I(i)=t(n+i)-t(n), i=1,2,3,4,5 ; 1 \leq n \leq N-i
$$

The maximum values of $I(i)$ represent the longest intervals in which $i$ FPs occurred. The cumulative
A $50 \mu \mathrm{VL} 250 \mathrm{~ms}$

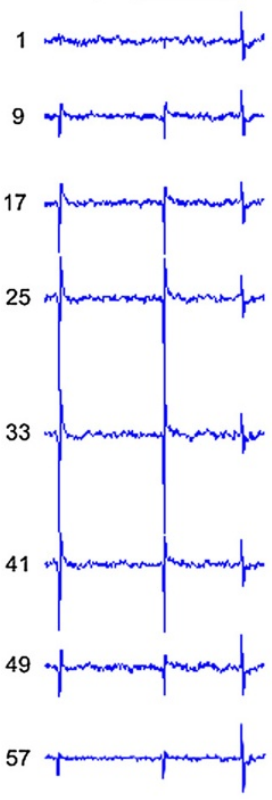

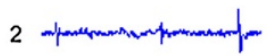

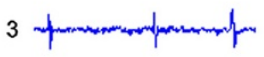

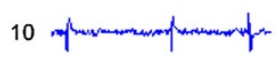

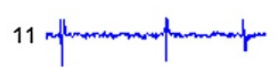

18

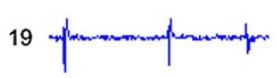

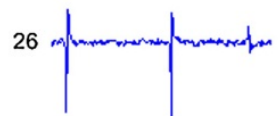
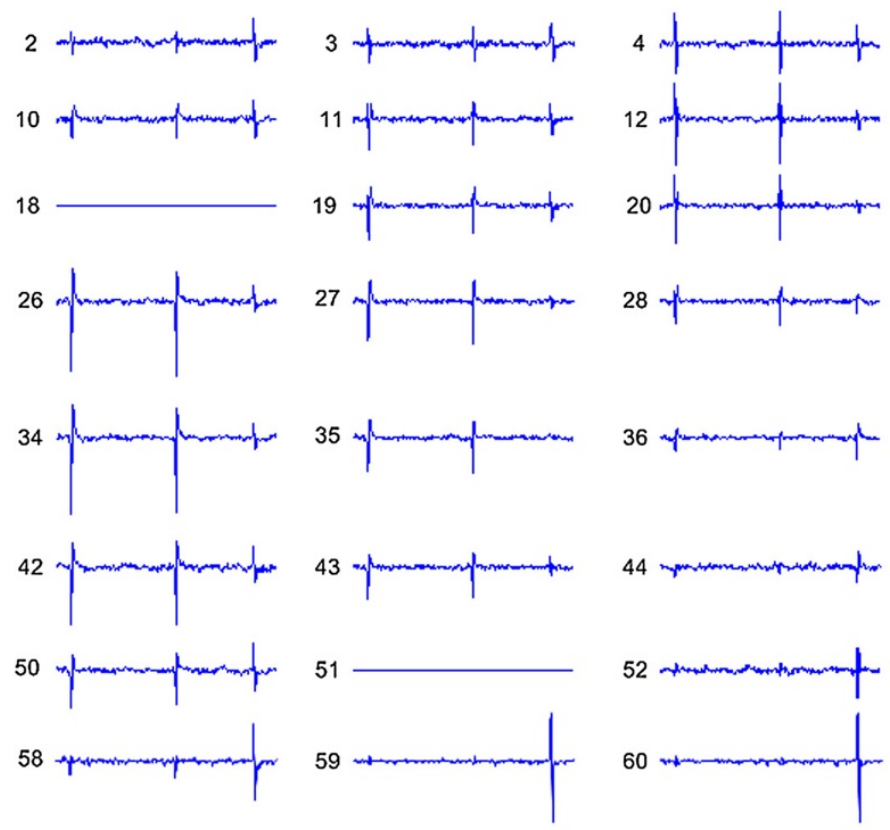

B

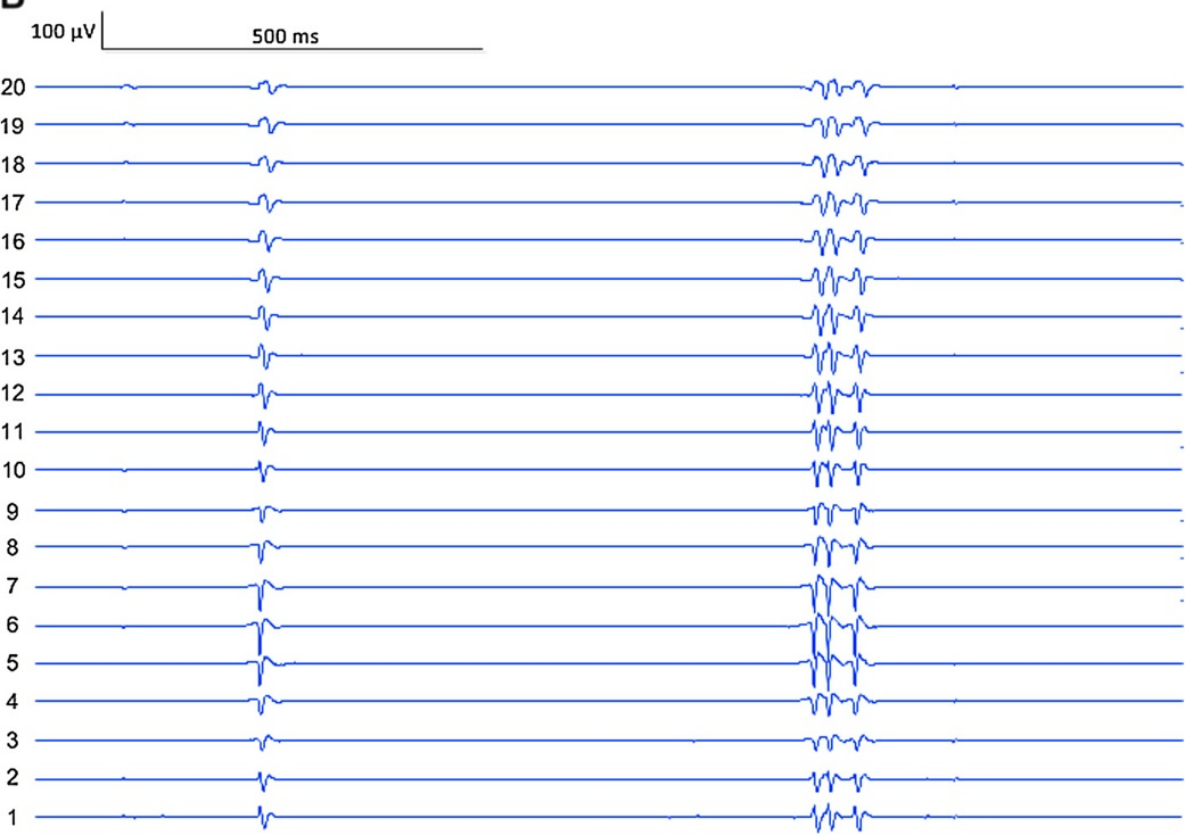

Figure 2 Examples of surface EMG signals recorded using the two electrode arrays. (A) Surface EMG signals recorded with the flexible electrode array from the thenar muscle. (B) Surface EMG signals recorded with the linear electrode array from the BB muscle. Channel 1 represents the most proximal channel. 
frequency distribution of $I(i)$ from the whole dataset was then constructed, allowing the probability of $i$ FPs occurring with respect to the duration of the recording to be calculated. In other words, after the electrode array has been placed and recording started, what will be the time range before $i$ FPs should have occurred?

\section{Results}

The duration of each recording of spontaneous muscle activity from the ALS subjects varied from $215 \mathrm{~s}$ to 764 s. Figure 2 shows an example of FP recordings using the two different electrode arrays from the thenar and $\mathrm{BB}$ muscles, respectively. FPs were recorded from all tested muscles. The overall FP rate was 3.5-139.0 FPs/ minute. The average skewness of the inter-FP-interval distribution was $1.06 \pm 0.71$, suggesting that the distribution of intervals between FPs was highly skewed with many short intervals and few long intervals. Across all muscles, the cumulative frequency distributions for one to five FPs are presented in Figure 3.

We show that the probability of recording a single FP using HD-SEMG was approximately 0.55 with a $20 \mathrm{~s}$ observation time and approximately 0.80 with a $40 \mathrm{~s}$ observation time. To record a single FP with a probability approaching unity, an observation time of up to $70 \mathrm{~s}$ may be needed. To record multiple FPs, a longer recording time is required as demonstrated in Figure 3. For example, the probability of recording five FPs approached approximately 0.95 with HD-SEMG recording for up to 2 minutes.

\section{Discussion}

Intramuscular needle electrodes have been routinely used in the clinical EMG examination for evaluation and diagnosis of neuromuscular diseases [6]. In recent years, multi-channel recordings have been developed in surface EMG technology using electrode arrays up to 128 channels [9-13]. Indeed, development of the multielectrode surface EMG montage represents an important research focus in several recent collaborative efforts sponsored by European Community (EC) [20]. HD-SEMG has been emerging as a potential clinical tool to supplement routine needle EMG. The spatial information of the electrode array can provide additional useful information (e.g., muscle fiber conduction velocity, motor unit territory, innervation zone localization) which is impractical or not possible to obtain with conventional needle electrodes [13-19].

For some specific evaluations, HD-SEMG may provide a potential substitute for routine needle EMG, while preserving diagnostic sensitivity and specificity. One example is to record FPs as demonstrated in this short report, as well as in previous studies [21-23]. Fasciculations are a characteristic and potentially diagnostic feature of ALS [24-27], although their precise role in establishing the diagnosis of ALS remains contentious based on routine needle EMG [3,4]. FPs may portend lower motoneuron dysfunction prior to clinical symptoms such as weakness or muscle atrophy, possibly before the onset of definite reinnervation changes in the motor unit action potentials $[6,22,24,25]$. Thus, enhanced detection of FPs may be of great importance for early diagnosis of ALS as well as better understanding their pathophysiology in this disorder.

In this brief report, we address the practical question of how long HD-SEMG should be used to record the tested muscle before being confident that no FPs are present. The method proposed by Mills [7] for testing with an intramuscular needle EMG electrode was utilized in this study. Considering the practicality in time needed for needle electrode recordings, Mills used a one minute recording epoch as the criterion in how long to monitor a muscle for FPs before it can be included in analysis. In other words, the muscle was excluded from analysis if it showed no FPs after 1 minute. In this study, up to $764 \mathrm{~s}$ spontaneous EMG signal was recorded for each muscle. These longer recording times avoided exclusion of muscles with long inter-fasciculation intervals. Hence we were able to calculate the probability function of observing one to five FPs with respect to recording duration. Although with a 2-dimensional surface electrode array it is feasible to differentiate FPs arising from different motor units using their waveform morphology

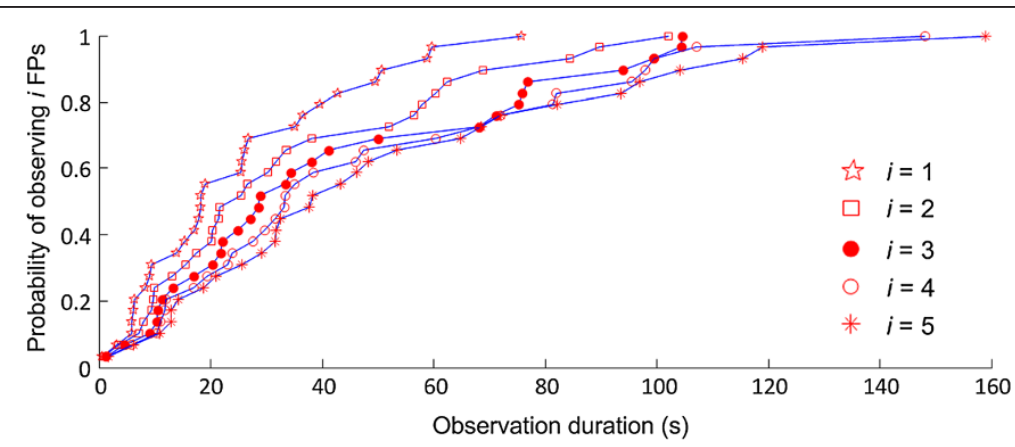

Figure 3 Cumulative probability of observing $i(i=1,2,3,4,5$, respectively) FPs with respect to observation duration of the HD-SEMG. 
to identify them with the added spatial resolution (particularly in perpendicular to muscle fibers) [28,29], our intent was to determine the longest interval between FPs regardless of waveform since these would have the same diagnostic significance. Our results confirm that HDSEMG offers a suitable and highly sensitive technique for capturing FPs. We acknowledge that simultaneous recordings with needle EMG and HD-SEMG from the same muscles would be necessary to directly compare sensitivity in FP detection between them. In addition, this study tested patients with Definite ALS or Probable ALS with Laboratory Support. It is presently unknown how the results may change for patients with Possible ALS or Suspected ALS.

It should be noted that the prediction of time for EMG surveillance in detecting FPs depends on the context of clinical situation [7]. With clear conventional evidence of denervation (i.e., fibrillation potentials) in a muscle, identification of a single FP may be sufficient to further support the possibility of ALS. Thus the longest time needed to observe such a muscle using HD-SEMG would be approximately $70 \mathrm{~s}$. Conversely, when clinical and conventional EMG abnormality is limited, a longer duration recording may be necessary. In this situation, the detection of FPs could have greater significance in determining electrophysiologic abnormality in a muscle. The current analysis demonstrates the longest time to be certain of recording one to five FPs using HD-SEMG. In most cases, however, FPs may be detected more quickly. After the suggested maximum recording time (approximately 70s for $1 \mathrm{FP} ; 120 \mathrm{~s}$ for $5 \mathrm{FPs}$ ), we feel confident that FPs are very likely to be absent in a tested muscle.

\section{Competing interests}

The authors declare that they have no competing interests.

\section{Authors' contributions}

PZ performed experiment design, data collection and analysis, interpretation of the results, and drafting of the manuscript. XL was involved in data collection and analysis. FJ was involved in data collection. WR was involved in interpretation of the results and revision of the manuscript. PB was involved in ALS subject recruitment, interpretation of the results, and revision of the manuscript. All authors read and approved the final manuscript.

\section{Acknowledgements}

This study was supported by the National Institute on Disability and Rehabilitation Research of the U.S. Department of Education (Grant H133G 090093) and the National Institutes of Health (Grant 2R24HD050821). Dr. Barkhaus also acknowledges support in part from the Dolores and Carroll Olson Fund of the Greater Milwaukee Foundation.

\section{Author details}

Sensory Motor Performance Program, Rehabilitation Institute of Chicago, 345 E. Superior St, Suite 1406, Chicago, IL 60611, USA. ²Department of Physical Medicine and Rehabilitation, Northwestern University, Chicago, IL, USA. ${ }^{3}$ Institute of Biomedical Engineering, University of Science and Technology of China, Hefei, China. ${ }^{4}$ Department of Bioengineering, University of Illinois at Chicago, Chicago, IL, USA. ${ }^{5}$ Department of Neurology, Medical College of Wisconsin and the Milwaukee Veterans Administration Medical Center, Milwaukee, WI, USA.
Received: 12 December 2011 Accepted: 4 October 2012

Published: 10 October 2012

\section{References}

1. Brooks BR, Miller RG, Swash M, et al for the World Federation of Neurology Group on Motor Neuron Diseases: El Escorial revisited: Revised criteria for the diagnosis of amyotrophic lateral sclerosis. Amyotroph Lateral Scler Other Motor Neuron Disord 2000, 1:293-299.

2. Nandedkar SD, Barkhaus PE, Sanders DB, et al: Some observations on fibrillations and positive sharp waves. Muscle Nerve 2000, 23:888-894.

3. de Carvalho M, Dengler R, Eisen A, England JD, Kaji R, Kimura J, Mills K, Mitsumoto H, Nodera H, Shefner J, Swash M: Electrodiagnostic criteria for diagnosis of ALS. Clin Neurophysiol 2008, 119(3):497-503.

4. Benatar M, Tandan R: The Awaji criteria for the diagnosis of amyotrophic lateral sclerosis: have we put the cart before the horse? Muscle Nerve 2011, 43:461-463.

5. de Carvalho M: Pathophysiological significance of fasciculations in the early diagnosis of ALS. Amyotroph Lateral Scler Other Motor Neuron Disord Suppl 2000, 1:S43-S46.

6. Barkhaus PE, Nandedkar SD: EMG Evaluation of the Motor Unit: "The Electrophysiologic Biopsy". In eMedicine Journal (Neurology) [serial online]. Edited by Quan D, Lorenzo NY, Lutsep HL: 2005. Available at http://www. emedicine.com/neuro/topic610.htm

7. Mills KR: Detecting fasciculations in amyotrophic lateral sclerosis: duration of observation required. J Neurol Neurosurg Psychiatry 2011, 82(5):549-551.

8. Howard RS, Murray NM: Surface EMG in the recording of fasciculations. Muscle Nerve 1992, 15:1240-1245.

9. Blok JH, van Dijk P, Drost G, Zwarts MJ, Stegeman DF: A high-density multichannel surface electromyography system for the characterization of single motor units. Rev Sci Instr 2002, 73:1887-1897.

10. Lapatki BG, van Dijk JP, Jonas IE, Zwarts MJ, Stegeman DF: A thin, flexible multielectrode grid for high-density surface EMG. J Appl Physiol 2004, 96:327-336

11. Pozzo M, Bottin A, Ferrabone R, Merletti R: Sixty-four channel wearable acquisition system for long-term surface electromyogram recording with electrode arrays. Med Biol Eng Comput 2004, 42:455-466.

12. Merletti R, Botter A, Troiano A, Merlo E, Minetto MA: Technology and instrumentation for detection and conditioning of the surface electromyographic signal: State of the art. Clin Biomech (Bristol, Avon) 2009, 24(2):122-134

13. Masuda T, Sadoyama T: Skeletal muscles from which the propagation of motor unit action potentials is detectable with a surface electrode array. Electroencephalogr Clin Neurophysiol 1987, 67:421-427.

14. Sun TY, Lin TS, Chen JJ: Multielectrode surface EMG for noninvasive estimation of motor unit size. Muscle Nerve 1999, 22:1063-1070.

15. Zwarts MJ, Stegeman DF: Multichannel surface EMG: basic aspects and clinical utility. Muscle Nerve 2003, 28:1-17.

16. Rau G, Schulte E, Disselhorst-Klug C: From cell to movement: to what answers does EMG really contribute? J Electromyogr Kinesiol 2004, 14:611-617.

17. Drost G, Stegeman DF, van Engelen BG, Zwarts MJ: Clinical applications of high-density surface EMG: a systematic review. J Electromyogr Kinesiol 2006, 16(6):586-602.

18. Merletti R, Holobar A, Farina D: Analysis of motor units with high-density surface electromyography. J Electromyogr Kinesiol 2008, 18(6):879-890. Epub 2008 Nov 11. Review.

19. Zhou P, Suresh NL, Rymer WZ: Surface electromyogram analysis of the direction of isometric torque generation by the first dorsal interosseous muscle. J Neural Eng 2011, 8(3):036028. Epub 2011 May 13.

20. Merletti R, Parker PA: Introduction, in Electromyography: physiology, engineering, and noninvasive applications. In Introduction, in Electromyography: physiology, engineering, and noninvasive applications. Edited by Merletti R, Parker PA. Hoboken, New Jersey: John Wiley \& Sons, Inc; 2004.

21. Drost G, Kleine BU, Stegeman DF, van Engelen BG, Zwarts MJ: Fasciculation potentials in high-density surface EMG. J Clin Neurophysiol 2007, 24:301-307.

22. Kleine BU, Stegeman DF, Schelhaas HJ, Zwarts MJ: Firing pattern of fasciculations in ALS: evidence for axonal and neuronal origin. Neurology 2008, 70:353-359. 
23. Zhou P, Barkhaus PE, Zhang X, Rymer WZ: Characterizing the complexity of spontaneous motor unit patterns of amyotrophic lateral sclerosis using approximate entropy. J Neural Eng 2011, 8(6):066010.

24. Rosenfeld J: Fasciculations without fibrillations: the dilemma of early diagnosis. Amyotroph Lateral Scler Other Motor Neuron Disord 2000, 1(Suppl 1):S53-S56.

25. de Carvalho M, Swash M: Fasciculation potentials: a study of amyotrophic lateral sclerosis and other neurogenic disorders. Muscle Nerve 1998, 21(3):336-344

26. Noto YI, Misawa S, Kanai K, Shibuya K, Isose S, Nasu S, Sekiguchi Y, Fujimaki Y, Nakagawa M, Kuwabara S: Awaji ALS criteria increase the diagnostic sensitivity in patients with bulbar onset. Clin Neurophysiol 2011. Epub ahead of print.

27. Mills KR: Characteristics of fasciculations in amyotrophic lateral sclerosis and the benign fasciculation syndrome. Brain 2010, 133(11):3458-3469. Epub 2010 Oct.

28. Farina D, Negro F, Gazzoni M, Enoka RM: Detecting the unique representation of motor-unit action potentials in the surface electromyogram. J Neurophysiol 2008, 100:1223-1233.

29. Kleine BU, van Dijk JP, Lapatki BG, Zwarts MJ, Stegeman DF: Using two-dimensional spatial information in decomposition of surface EMG signals. J Electromyogr Kinesiol 2007, 17:535-548.

doi:10.1186/1743-0003-9-78

Cite this article as: Zhou et al:: Duration of observation required in detecting fasciculation potentials in amyotrophic lateral sclerosis using high-density surface EMG. Journal of NeuroEngineering and Rehabilitation 2012 9:78.

\section{Submit your next manuscript to BioMed Central and take full advantage of:}

- Convenient online submission

- Thorough peer review

- No space constraints or color figure charges

- Immediate publication on acceptance

- Inclusion in PubMed, CAS, Scopus and Google Scholar

- Research which is freely available for redistribution 